\title{
Special issue on emerging trends in soft computing: memetic algorithms
}

\author{
Yew-Soon Ong • Meng-Hiot Lim • Ferrante Neri • \\ Hisao Ishibuchi
}

Published online: 26 July 2008

(C) Springer-Verlag 2008

\section{Introduction}

In the last few decades, evolutionary algorithms (EAs) have gradually established a strong foothold as powerful search methods in many complex disciplines ranging from arts \& design, business \& finance to science $\&$ engineering. Although well established as credible and powerful search tools, researchers in this area are now facing new challenges of increasing computational needs in today's applications. The popularity and success of EAs lie in the ease of implementation, generality of the concept and their ability to converge close to the global optimal design. Nevertheless, traditional EAs generally suffer from excessively slow convergence to locate a precise enough solution efficiently due to the lack of individual learning capabilities. This often limits the practicality of EAs on many large-scale real world problems where the computational time is a crucial consideration. One of the recent most important and successful areas of research that has spawned from evolutionary computations

Y.-S. Ong ( $\varangle)$

School of Computer Engineering, Nanyang Technological

University, Singapore, Singapore

e-mail: asysong@ntu.edu.sg

\section{M.-H. Lim}

School of Electrical and Electronic Engineering,

Nanyang Technological University, Singapore, Singapore

e-mail: emhlim@ntu.edu.sg

F. Neri

Department of Mathematical Information Technology,

University of Jyväskylä, Jyväskylä, Finland

e-mail: neferran@cc.jyu.fi

\section{H. Ishibuchi}

Department of Computer Science and Intelligent Systems, Osaka Prefecture University, Osaka, Japan

e-mail: hisaoi@cs.osakafu-u.ac.jp is the methodology known as Memetic algorithms (MAs). MAs are population-based meta-heuristic search approaches that are inspired by Neo-Darwinian's principles of natural evolution and Dawkins' notion of a meme defined as a unit of cultural evolution that is capable of performing individual learning, for example, in the form of local refinements. The metaphorical parallels, on the one hand, Darwinian evolution and, on the other hand, between memes and domain specific heuristics are captured within MAs, thus rendering a methodology that balances well between generality and problem-specificity. It has shown to be successful and popular for search problems whereby high-quality solutions can be attained much more efficiently.

This special issue containing 10 papers reflects the recent state-of-the-art advances in the research \& development of MAs. Particularly, it serves to present the current trends in sophisticated MA methodologies and frameworks, report the success of the technology to unexplored real-world problem domains, as well as to assist in the identification of new research directions in this field of MA.

\section{Structure of the special issue}

The entire special issue is loosely grouped into two categories: (1) Methodologies and Frameworks and (2) DomainSpecific Applications. Part I consists of five papers, dealing primarily with new design methodologies and frameworks of MAs while the latter concentrates on specialized MAs designed for specific applications.

\subsection{Memetic algorithm methodologies \& frameworks}

The papers by Barkat Ullah et al., Wang et al., Tenne and Ishibuchi et al. represent well the latest trends in the 
development of MA methodologies \& frameworks that are designed for solving well-established complex areas pertaining to general constrained non-linear programming, dynamic and uncertain environments, computationally expensive problems and multi-objectivity. At the same time, the papers by Barkat Ullah et al. and Caponio et al. also reported new concepts and methodologies for adapting the choice of memes in MA. One clear trend that has emerged from these papers is the general acceptance of memetics as a viable and successful concept to a plethora of new emerging areas in soft computing. This is indicative of the emergence of MA as a technique that transcends beyond the classical notion of evolution that tends to focus on the solution structures or representations. In essence, MA is now the embodiment of evolution with a much broadened scope.

The first paper, "AMA: A new approach for solving constrained real-valued optimization problems" by Barkat Ullah et al. focused on real-valued constrained problems and proposed an agent based MA where chromosomes exists as individual autonomous agents that possess the ability to adaptively select appropriate solver(s) from a pool containing multiple pre-defined solvers. The second paper, "A memetic algorithm with adaptive hill climbing strategy for dynamic optimization problems" by Wang et al. described a Memetic framework that adapts the hill-climbing local search method for solving general dynamic optimization problems. In their proposed work, two diversity preservation approaches were introduced to highlight the importance of avoiding pre-mature convergence when designing MAs for solving dynamic and uncertain problems. The third paper, "A framework for memetic optimization using variable global and local surrogate models" by Tenne proposed a novel MA methodology that employs both local and global surrogate assisted models for solving optimization problems that are plagued with computationally expensive objective function(s). In their work, the proposed algorithm was also demonstrated to perform well on a realistic airfoil design problem. The fourth paper, "Use of biased neighborhood structures in multi-objective memetic algorithms" by Ishibuchi et al. highlights the importance of memetics within the field of multi-objective evolutionary search. In their work, the issues relating to biased neighborhood structure local search in multi-objective MAs was comprehensively investigated in the context of $0 / 1$ knapsack and flowshop scheduling problems. Under a biased neighborhood structure, each neighbor of a current solution has a different probability to be sampled in the local search. The fifth paper, "Super-fit control adaptation in memetic differential evolution frameworks" by Caponio et al. described a novel MA composed of a Differential Evolution framework and a set of local searchers adaptively coordinated by means of a fitness diversity logic which measures the quality of the best solution (super-fit) with respect to the other individuals.

\subsection{Domain-specific applications}

Five papers reporting novel dedicated MAs crafted to solve domain-specific problems efficiently are also included in the present issue to demonstrate the continuing success of MA in practice and in new unexplored application domains.

Goh et al. in "A hybrid evolutionary approach for heterogeneous multiprocessor scheduling" reported a tailored MA for assignment of tasks in a heterogeneous multiprocessor environment with interdependencies. Qian et al. in "Multiobjective no-wait flow-shop scheduling with a MA based on differential evolution" described a MA composed of differential evolution and local searchers for solving multi-objective no-wait flow-shop scheduling problems. The paper entitled "A Case Study of MAs for Constraint Optimization" by Ozcan et al., performed an empirical investigation of several MAs that is a hybridization of genetic algorithm and local hill climber for solving multidimensional $0-1$ knapsack problems. The paper "Performance evaluation of memetic approaches in 3D reconstruction of forensic objects" by Santamaría et al., described a MA containing multiple local solvers to solve an image registration problem in the field of Anthropology. The capability of MAs for 3D reconstruction of forensic objects is analyzed extensively in their work. Last but not least, Dalila Boughaci et al. in "Memetic Algorithm for Optimal Winner Determination Problem" reported a new MA for the optimal winner determination problem in combinatorial auctions.

Acknowledgments We would like to thank all the authors that submitted papers to this special issue and we would also like to acknowledge the stupendous efforts of the numerous referees that helped in reviewing and selecting these papers. We would like to give special thanks to Professor Vincenzo Loia and his production team for their continuous support that made this special issue a great success. 